\title{
TOOLS FOR ASSESSING THE COMMUNICATIVE COMPETENCE OF UNDERGRADUATE ESP FOR BUSINESS STUDENTS AT THE SOUTH EAST EUROPEAN UNIVERSITY
}

\begin{abstract}
The interest in the concept of communicative competence among ESP instructors has been continuously growing. Parallel to this, there has been a heightened awareness of the need for reliable assessment tools to evaluate the actual use of the target language and not only the surface knowledge of vocabulary and grammar rules, which are in most cases easily assessed through multiple-choice tests.

Developing students' communicative competence is one of the desired learning outcomes of the ESP for Business courses offered at the South East European University (SEEU). This paper, offers an insight into the use of mock job interviews, role plays based on case studies and informative presentations as tools for assessing the communicative competence of undergraduate ESP for business students at SEEU.
\end{abstract}

Key words: Communicative competence, assessment, Business English, SEEU

\section{Introduction}

Developing students' ability to use English effectively in the increasingly globalized world of work is an objective shared by all courses of ESP for Business, and the courses offered at the South East European University (SEEU) are no exception.

This paper aims to briefly summarize what is meant by communicative competence and present its constituting components. It also draws attention to the fact that non-verbal communication is unjustly neglected and needs to be paid more attention to. Moreover, it offers an insight into SEEU experience and current approach towards assessment of students' communicative competence through assignments which resemble task-based language learning. This paper should be perceived as a starting point in the development of further assessment tools. Hopefully, it will inspire new ideas about how to approach the assessment of communicative competence of undergraduate students of ESP for Business.

\footnotetext{
* Language Centre, South East European University, Ilindenska n. 335, 1200 Tetovo, North Macedonia; e-mail: m.marjanovic@seeu.edu.mk
} 


\section{Communicative competence}

The overall ability of people to reach their goals in social life depends to a large extent on their communicative competence, a notion which is not easy to define primarily because of the complexity of communication as a process, the wide variety of related cognitive and social abilities as well as the huge situational variability. The concept of 'communicative competence' was introduced by the sociolinguist Dell Hymes in the 1960s to emphasize the fact that the knowledge of grammatical rules is not sufficient for speaking a language and for effective communication in it.

Taking into consideration that fact that communication is always goals-oriented it can freely be concluded that communicative competence is inevitably related to the concepts of effectiveness and appropriateness. These two concepts are the central criteria according to which communicative competence can be defined as the extent to which the speaker manages to achieve the preferred outcomes and goals set in accordance with the situational conditions. Effectiveness may refer to the ability to convey meaning or to infer a speaker's meaning, whereas appropriateness refers to the effort made in order to avoid breaking the social and interpersonal standards, norms and expectations.

According to Canale (2013), communicative competence is a part of real communication and is in fact a collection of language knowledge and skill (or ability) to apply the knowledge when interacting in realistic situations. The four areas of knowledge and skill which constitute Canale's model of communicative competence are: grammatical, sociolinguistic, discourse and strategic competence.

The grammatical or formal competence refers to the concept of linguistic competence used by Chomsky (1965) which indicates the native speaker's knowledge of syntactic, lexical, morphological and phonological features of the language which results in producing well-formed words and sentences, or, in other words, results in accurate performance. The sociolinguistic competence deals with the rules referring to the social context in which the language is used. It includes the role of the participants in a certain interaction, their social status, the information they share and the function of the interaction itself. The social context is created by culture specific norms, values, beliefs and behavioural patterns of a culture. In order to use language appropriately, attention should be paid to the culture-specific context. The discourse competence (Canale 2013) is in fact the ability to use connected sentences or utterances as a meaningful whole in context. Both general knowledge of the world as well as knowledge about a particular context are used to link the ideas expressed since ideas which are not adequately linked will most likely lead to erroneous meaning being inferred. Strategic competence is defined as the ability to cope with an authentic 
communicative situation and keep the communication channel open. In order to achieve this, knowledge of communicative strategies is required. The communicative strategies are needed to compensate for imperfect knowledge of rules or factors such as inattention or distraction which limits the application of rules. This refers to strategies about what to do in case you cannot think of the right word or when it is obvious that the message you have sent was misunderstood.

In the Common European Framework of Reference (2016), communicative competence is considered to be comprised of three separate components: linguistic, sociolinguistic and pragmatic competence, each of which is comprised of knowledge, skills and know-how. The linguistic competence, which includes lexical, phonological, syntactical knowledge and skills, is related not only to the range and quality of knowledge but also to its cognitive organization and the way this knowledge is stored as well as its accessibility (i.e., activation, recall and availability). The cognitive organisation of vocabulary, storing of expressions and the accessibility of knowledge in general will vary from one individual to another depending, amongst other things, on the cultural features of the community in which the individual has been socialised and where his or her learning has occurred. The main components of linguistic competence defined as knowledge of, and ability to use the formal resources from which well-formed, meaningful messages may be assembled and formulated are lexical competence, grammatical competence, semantic competence, phonological competence, orthographic competence and orthoepic competence.

The sociolinguistic competence (CEFR 2019) refers to the sociocultural conditions of language use or, more precisely, it is concerned with the knowledge and skills required to deal with the social dimension of language use since language is a sociocultural phenomenon. Sociolinguistic competence focuses on linguistic markers of social relations, politeness conventions, expressions of folk-wisdom, register differences, and dialect and accent.

The pragmatic competence is concerned with the functional use of linguistic resources i.e., it is concerned with the knowledge of the principles according to which messages are: organised, structured and arranged which is in fact 'discourse competence'; the principles used to perform communicative functions which is 'functional competence'; as well as the principles according to which messages are sequenced according to interactional and transactional schemata or the so called 'design competence'.

The concept of communicative competence is of theoretical and also practical relevance; however, practical needs have always been a driving force for the development of the concept. Achieving communicative competence requires a number of processes and factors working together. The individual importance of each of the 
factors involved varies dependent on the particular communicative situation involved. A large number of specific theories account for different aspects of communicative competence. However, despite such diversity, they complement each other and converge in fundamental issues.

\section{Nonverbal communication}

Nonverbal communication plays a key role in an individual's overall communicative competence. The efficiency in encoding the needs and signalling attributes possessed influences the ability to achieve the set goals to a great extent. Equally important is the ability to precisely decode signals received form others and respond appropriately. In a word, nonverbal behaviour has a significant influence on the interpersonal exchange dynamics.

Communication is primarily associated with what people say or send as message and/or e-mail, i.e., it is associated with the contents exchanged when conveying the message. However, communication is by far more inclusive. Hargie (2017) draws attention to studies which show that the large majority of interaction time during a day is not taken up by speech but by nonverbal communication. Contrary to this, in general, we are more aware of the actual words we say rather than the nonverbal accompaniment of what is said. In other words, we carefully monitor what is said in order to achieve the desired effect but this does not apply to how we are saying it. With reference to the contribution of verbal and nonverbal element in the overall communication, Mehrabian (2007, in Hargie 2017) estimates that overall communication is made up of 55\% body language, $38 \%$ nonverbal aspects of speech or paralanguage and only $7 \%$ verbal content. It comes as a surprise that what we say contributes mere $7 \%$ to the overall message received. However, "nonverbal communication does not have to be shown to be more important than the verbal in order to be significant" (Hargie 2017: 45).

Numerous pieces of research conducted suggest that nonverbal behaviour, such as facial expressions and body movement, convey a lot more information about the speaker's thoughts and feelings since nonverbal communication is by far more universal compared to language. Research also confirms that in cases of conflicting verbal and nonverbal signals, nonverbal signals are considered to be more reliable. Similarly, when a verbally expressed message is unclear, the interlocutor relies exclusively on nonverbal signs.

Nevertheless, it should not be automatically assumed that nonverbal communication is similar in all cultures. Being unaware of the nuances in cultural diversity can easily result in miscommunication and interaction breakdown. This can also be a result of failure to use the right words. Hamilton (2014, in Hargie 2017) 
observes that a common mistake among business people travelling abroad is their assumption that business people there will more or less observe the same conventions they themselves are accustomed to.

Taking all this into consideration, it is more than obvious that the overall communicative competence of undergraduate Business English students can be considerably improved by increasing the awareness of the role of nonverbal behaviour in communication, especially during negotiations and team work when nonverbal exchange is a crucial indicator whether a certain team member is considered to contribute to the team work or not.

Presentations done in class are an ideal option for practising nonverbal communication through establishing appropriate eye contact with the audience. Students are reminded to scan the audience first and then establish eye contact lasting for approximately five seconds with individual audience members. Students are also reminded that instead of standing stiff in one spot, they should move around a bit.

During mock job interviews and group role plays, nonverbal signs indicate if the interlocutor is attentively listening to what is being said and is signalling genuine interest or lack of interest in what is being said. A simple nod of the head is an important visual sign indicating attention, interest and active information processing.

\section{Tools for teaching and assessing communicative competence employed at SEEU}

Since "the ultimate purpose of language learning is language use" (Hutchinson and Waters 1987: 109), what all ESP for Business courses should have in common is achieving the shared goal of developing students' ability to effectively and appropriately use the target language in the increasingly globalised world of work. In order to achieve this aim, the ESP for Business courses should incorporate the key features of real-life business communication.

In a study devoted to the types of tasks performed by professionals in international business centres in English as the main language of business communication, Evans (2013: 291) identifies several key characteristics of real-world business communication that all ESP practitioners should consider when teaching and assessing general-purpose Business English courses:

- the pace, pressure and unpredictability of business communication;

- emphasis on problem posing and solving;

- the central position taken by intertextuality and collaboration;

- the interplay between written and spoken discourses;

- the importance of precision and concision. 
The incorporation of the above listed key characteristics of real-world business communication in the design of tasks included in ESP for business courses would undoubtedly result in a considerable reduction of the gap separating the language classroom and the office.

In order to help students become communicatively competent, they should be enabled to personally experience the use of the target language they are learning in authentic communicative situations and develop the necessary skills required for applying the knowledge about the language they possess.

The three specific tasks employed in the courses of ESP for Business offered at the SEEU intended for the development and assessment of oral communicative competence are: informative presentations, mock job interviews and simulations of role plays based on case studies. The focus is on these activities mainly because they enable the incorporation of the key characteristics of real-world business communication, which automatically enables students to develop oral business communication skills and produce the language that they shall need in their future work posts.

\subsection{Informative presentation}

As a grading requirement, ESP for business students at SEEU are asked to do research about a company or a business-related topic of their own choice and present the findings in a 10-minute informative presentation accompanied by PowerPoint or Prezi. Informative presentations are an excellent example of an activity in which the border line between language and skills needed to communicate effectively is blurred. ESP teachers who actually evaluate language only do their students a really bad favour. Although the presentation is done primarily as part of a language course in which the main aim is to teach and learn the target language and the audience is primarily interested in the message and content of the presentation, the foreign language taught goes hand in hand with the skills needed to present effectively. Consequently, balance between language and skill should be achieved and reflected not only in teaching and developing effective presentation skills but also in establishing grading criteria.

As part of the teaching process, students who attend ESP for business at SEEU are asked to listen to a presentation given by a famous business leader. They are provided with the transcript of the speech and asked to predict the exact places where an intentional pause shall be made, where the accent will fall, where the pitch is most likely to rise or fall and afterwards compare their prediction with the actual presentation.

Alternatively, a useful strategy often used by ESP teachers at SEEU for gaining credibility for developing the key business skill of presenting effectively is making a 
formal, well- structured presentation introducing the course of ESP for business at the beginning of the semester and asking students to analyse the presentation and identify strategies for opening the presentation, using discourse markers, adequately using visuals and all other aspects considered important for giving an effective presentation.

Students are asked to prepare and practise at home and finally present in class in front of the teacher and other students. With the students' prior consent the presentations are filmed and consequently, depending on the students' preferences, analysed in oneto-one conferencing sessions or whole class sessions.

The informative presentation accounts for $15 \%$ of the final grade and its assessment breakdown is as follows:

- teacher evaluation rubric $-10 \%$ of the final grade

- post-presentation self-assessment - 5\% of the final grade

- peer assessment rubric on polleverywhere.com

The presentation evaluation rubric used by the teacher consists of five categories with maximum 2 points each: organization \& comprehensibility, time management, eye-contact and elocution, language, and overall task achievement.

While one student is presenting the other students in the class along with the teacher form the audience who listen attentively to the presentation and ideally actively participate in a follow-up discussion on the company/topic presented. After the discussion, students forming the audience are instructed to provide constructive criticism, offer suggestions for improvement and anonymously evaluate the presenter on https://www.polleverywhere.com/ against a list of criteria which focus on:

- Supplementary visuals (laptop, projector, pointer, board, handouts, etc.)

- Eye contact, body language, humour, etc.

- Delivery (clarity, pronunciation, pauses, intonation, etc.)

- Language use (accuracy, precision, appropriateness, vocabulary, etc.).

After the presentation, the student presenters are asked to reflect on the task and complete the self-evaluation for which they are given a week, but are advised to do while the impressions are still fresh. In the post-presentation feedback the students are asked to focus and briefly elaborate on three aspects:

- the pre-presentation or preparatory stage (the way they did the research, difficulties and obstacles that they might have faced and the way they overcame them);

- performance or the actual presentation done in class (whether everything went according to plan or there were some unanticipated problems);

- lesson learned (what would be done differently next time they present). 
Developing reflective language learning attitude is a pre-requisite for becoming an autonomous life-long language learner. Hopefully, in the future when asked to present, the students will recall the strengths and weaknesses of the presentation done in class.

\subsection{Mock job interviews}

For mock job interviews depending on the number of students in a section, students are ideally assigned both the role of an applicant and employer. However, in most cases due to class size and time restraints, students are randomly assigned one of the roles. As is the case with the informative presentations, with prior students' consent the interviews are filmed and consequently analysed together by the entire class or in one-to-one conferencing sessions.

After reading a number of selected articles on the topic of job hunting, watching selected videos on the topic, prioritising the major DOs and DON'Ts of job interviews, anticipating most likely questions and discussing the most appropriate answers to them, the interviews are actually acted out in front of the teacher and the classmates. According to the number of students per section, one to three students are on the interview panel and one or two students are applying for a job. For the sake of authenticity, students are applying to real job adverts for employment or internship provided by the SEEU Career Centre. When two students are applying for the same post, the interviewers have to say who gets the job and elaborate on the choice made. Using a grading rubric, the teacher evaluates each individual student and students self-evaluate focusing on the preparation for the interview, the actual performance in class and the lesson learnt. Classmates have a chance to anonymously provide constructive criticism, other alternatives and suggestions for improvement on www.pollevrywhere.com.

\subsection{Role plays based on case studies}

When designing, planning and setting role plays based on case studies in the ESP for BA courses at SEEU, students are always assigned clearly defined roles in a particular business context and they are expected to quickly and collaboratively investigate and resolve certain problems. During the role play, students process and produce interdependent text types, such as brief reading passages, emails and reports, and participate in speech events, such as simulated meetings, telephone conversations or negotiations. All this corresponds to task-based language teaching where the emphasis is on purposeful communication and various skills integration. Special attention is being placed on the effort to ensure that the pace, pressure, and unpredictability of the business world communication is reflected, making the students 
communicate fast and spontaneously but at the same time precisely and concisely in order to accomplish the task assigned. Strict time limits are imposed and information is revealed and added at specific stages during the role plays which in is most cases are telephone conversations, meetings, negotiations or even group debates. A problem is posed to which the students are expected to find, propose and negotiate solutions.

The background information and roles are provided by the case studies which are the final section of each unit in the textbook Business Result intermediate by Oxford University Press (2012) used at SEEU. The background information is usually presented through a brief reading passage.

Although in role plays students are assigned specific roles which determine not only their actions but dictate their opinion as well, one of their major benefits is the information gap - none of the participants has all the relevant information, which creates a real need for communication. What is more, additional information is dosed and timely added, which creates the need for the students to make on-the-spot decisions about the direction of the communication. Having prescribed roles which dictate and direct options is extremely useful for pre-experienced students since a large portion of the information needed is readily served to them.

One of the disadvantages of role plays is the necessary preparation time. Unless properly prepared, the role play will be frequently interrupted by students stopping to check out what comes next and what they are supposed to say next.

With respect to the actual assessment of role plays, apart from the evaluation rubric used by the teacher for every individual student, the students themselves get to evaluate the individual contribution of each group member separately and reflect on their own contribution in the group work.

Simulations, such as presentations and interviews, are considerably different from role plays. They are activities in which students are themselves and (ideally) simulate what they would actually do in real life. The aim of a simulation is to turn the classroom into a so-called safe zone, where students would experiment and practise so when faced with a same or similar context in real life they could freely say: "Aha, I've been there and I've done that." For a simulation to be successful a real reason to communicate is needed, otherwise students will quickly switch to using their native language especially in a monolingual class. A simulation requires pretending in order for students to become completely immersed in the role play and able to forget that they are just practising English in a classroom. A solid indicator that a simulation is successful is the continuation of the discussion after the lesson. At SEEU, for instance, Google Classroom is always used as an option for continuing the discussion after class.

Apart from informative presentations (followed by asking and answering questions and discussing the topic presented with the audience comprised of the 
teacher and classmates) and job interviews, another frequently used role play which can easily been turned into a simulation, is meetings in which students are asked to present information, discuss, and defend their option in order to reach a mutually acceptable solution to the problem posed, which is a frequent scenario in real-life business contexts.

During simulations and role plays, the teacher monitors closely, noting weaknesses in the language use and offering suggestions for possible improvement. Good examples should always be pointed out. In some cases, such as during presentations for instance, there is no reason whatsoever for the teacher not to take an active part as an audience member.

The preparation for the tools utilised for teaching and assessing the undergraduate ESP for business students' communicative competence should include:

- determining the aim of the activity and ensuring its relevance to students' real needs;

- ensuring a logical flow of planned activities;

- including a variety of realistic, challenging and interesting activities;

- enabling active participation of all students;

- enabling students to use their present knowledge and experience;

- if the task is focused on problem solving, accepting more than one possible solution;

- making sure students understand the situation being simulated or role played, the reason behind it as well as its goals;

- planning enough preparation time (very frequently the preparatory stage is longer than the simulation or the role play itself);

- providing students with feedback on their participation and performance in the simulation or role play and asking them for suggestions for possible alternative designs of the tasks in other to improve them.

In all simulations and role play tasks done in class the teacher has to make sure students experience repeated success and accomplishment in order to gain confidence in their ability to communicate.

The results from the annual student course evaluations conducted at SEEU indicate that undergraduate students of ESP for business consider informative presentations, mock job interviews and role plays based on case studies as appropriate and useful instruments for teaching and assessing communicative competence. This is a strong enough argument for us, ESP for BA teachers, to continue implementing them while continuously searching for other, even more appropriate, assessment tools for evaluating students' communicative competence. 


\section{Conclusion}

In order to help pre-experienced undergraduate students of ESP for business become communicatively competent, they need to be enabled to personally experience the use of the language they are learning and they need to be assisted in developing skills for applying the knowledge about the language they possess in authentic communicative acts. Although the in-class informative presentations, mock job interviews and role plays done in the classroom shall never be able to exactly replicate the conditions and complexities of the workplace, they nevertheless do "help to narrow the office/classroom divide and provide students with a more practical and engaging learning experience" (Evans 2013: 292) they could rely on in order to survive their first employment.

Assessing the communicative competence of undergraduate students of ESP for Business using the tools suggested is indeed a very time-consuming process, but the results are worthwhile due to the fact that it gives a more holistic, meaningful measure of the students' communicative skills compared to the evaluation based on conventional multiple-choice testing. These assessment instruments enable both teachers and students to identify various aspects of the oral communication which need to be addressed in order to become more communicatively competent. Parallel to this, as a bonus end result, students acquire a greater degree of confidence in speaking the language, which in turn has a positive accumulative effect on their overall ability to speak the language.

\section{References}

Canale, M. (2013). From Communicative Competence to Communicative Language Pedagogy. In: J. C. Richards and R. W. Schmidt (eds.), Language and Communication, London and New York: Routledge, 2-28.

Chomsky, N. (1965). Aspects of the Theory of Syntax. Cambridge: MIT Press.

CEFR: Council of Europe. 2019. Common European Framework of Reference for Languages: Learning, Teaching, Assessment. (29 May 2019)

$<$ http://www.coe.int/t/dg4/linguistic/Source/Framework_EN.pdf $>$.

Dudley-Evans, T. and M. St John (1998). Developments in English for Specific Purposes: A Multi-disciplinary Approach. Cambridge: Cambridge University Press.

Evans, S. (2013). Designing Tasks for the Business English Classroom. ELT Journal, $67.3,281-293$. 
Evans, S. (2013). A case study of Business English Teaching Materials adaptations from SFL - ESP perspectives. (29 May 2019) <https://www.researchgate.net/ publication/319561426>.

Hargie, O. (2017). Skilled Interpersonal Communication Research, Theory and Practice. London and New York: Routledge.

Hughes, J. and J. Naunton (2012). Business Result, Intermediate. Oxford: Oxford University Press.

Hutchinson, T. and A. Waters (1987). English for Specific Purposes. Cambridge: Cambridge University Press. 